DR. AGUSTIN IBANEZ (Orcid ID : 0000-0001-6758-5101)

Article type : Special Issue Article

\title{
Heart-brain interactions during social and cognitive stress in hypertensive disease: a multidimensional approach
}

Running Title: Multidimensional markers of stress in hypertension

Authors: Agustina Legaz ${ }^{1,2,3}$, Adrián Yoris ${ }^{1,4}$, Lucas Sedeño ${ }^{1}$, Sofía Abrevaya ${ }^{1,4}$, Miguel Martorel1 ${ }^{1,4}$, Florencia Alifano ${ }^{1,4}$, Adolfo M. García ${ }^{1,2,5,6, *}$, Agustín Ibañez 1, 2,6,7,8, *

\section{Affiliations:}

${ }^{1}$ National Scientific and Technical Research Council (CONICET), (C1126AAB), Buenos Aires, Argentina

${ }^{2}$ Cognitive Neuroscience Center (CNC), Universidad de San Andrés, Buenos Aires, Argentina

${ }^{3}$ Universidad Nacional de Córdoba. Facultad de Psicología, Córdoba, Argentina

${ }^{4}$ Institute of Cognitive and Translational Neuroscience (INCYT), INECO Foundation, Favaloro University, CONICET, Buenos Aires, Argentina

${ }^{5}$ Faculty of Education, National University of Cuyo, Mendoza, Argentina

${ }^{6}$ Global Brain Health Institute (GBHI), University of California San Francisco (UCSF), San

Francisco, United States

${ }^{7}$ Universidad Autónoma del Caribe, Barranquilla, Colombia

${ }^{8}$ Center for Social and Cognitive Neuroscience (CSCN), School of Psychology, Universidad Adolfo Ibáñez, Santiago, Chile

This article has been accepted for publication and undergone full peer review but has not been through the copyediting, typesetting, pagination and proofreading process, which may lead to differences between this version and the Version of Record. Please cite this article as doi: 10.1111/ejn.14979

This article is protected by copyright. All rights reserved 
* Corresponding Authors: A. Ibáñez (agustin.ibanez@gbhi.org) and A. M. García (adolfomartingarcia@gmail.com), Cognitive Neuroscience Center (CNC), Universidad de San Andrés, Vito Dumas 284, B1644BID Victoria, Buenos Aires. Phone/Fax: (+54) 11 4725-7000.

Number of pages: 37 pages; Figures: 1; Tables: 4.

Number of words in the whole manuscript: 9981 words.

Number of words in the main text: 5514 words.

Number of words in the Abstract: 249 words.

Keywords: Heart rate variability, hypertension, psychosocial stress.

\section{Abstract}

Hypertensive disease (HTD), a prominent risk factor for cardiovascular and cerebrovascular diseases, is characterized by elevated stress-proneness. Since stress levels are underpinned by both cardiac and neural factors, multidimensional insights are required to robustly understand their disruption in HTD. Yet, despite their crucial relevance, heart rate variability (HRV) and multimodal neurocognitive markers of stress in HTD remain controversial and unexplored, respectively. To bridge this gap, we studied cardiodynamic as well as electrophysiological and neuroanatomical measures of stress in HTD patients and healthy controls. Both groups performed the Trier Social Stress Test (TSST), a validated stress-inducing task comprising a baseline and a mental stress period. During both stages, we assessed a sensitive HRV parameter (the low frequency/high frequency [LF/HF ratio]) and an online neurophysiological measure (the heartbeatevoked potential $[\mathrm{HEP}])$. Also, we obtained neuroanatomical data via voxel-based morphometry (VBM) for correlation with online markers. Relative to controls, HTD patients exhibited increased $\mathrm{LF} / \mathrm{HF}$ ratio and greater HEP modulations during baseline, reduced changes between baseline and stress periods, and lack of significant stress-related HRV modulations associated with the grey matter volume of putative frontrostriatal regions. Briefly, HTD patients presented signs of stressrelated autonomic imbalance, reflected in a potential basal stress overload and a lack of responsiveness to acute psychosocial stress, accompanied by neurophysiological and neuroanatomical alterations. These multimodal insights underscore the relevance of neurocognitive data for developing innovations in the characterization, prognosis, and treatment of 
HTD and other conditions with autonomic imbalance. More generally, these findings may offer new insights into heart-brain interactions.

\section{Introduction}

Hypertensive disease (HTD), defined by high blood pressure (Unger et al., 2020), ranks among the highest risk factors for disabling cardiovascular and cerebrovascular diseases (Ruediger et al., 2004; Thayer et al., 2010). Moreover, this disorder is typified by elevated stress-proneness (Wirtz et al., 2006; Liu et al., 2017a), a factor that also undermines cardiovascular function (Cohen et al., 2007; McEwen, 2007) and further compromises the patients' physical and mental health (Wiener et al., 2020). Since stress levels are underpinned by both cardiac and neural mechanisms (Gianaros \& Sheu, 2009; Allen et al., 2014), multidimensional insights are required to robustly understand their disruption in HTD. Measures of heart rate variability (HRV) are robust markers of mental stress (Taelman et al., 2009; Allen et al., 2014; Kim et al., 2018), especially if complemented with neurocognitive assessments (MacKinnon et al., 2013; Wei et al., 2018). However, HRV studies on HTD are controversial due to methodological inconsistencies and limitations (including suboptimal methods to induce stress, unreliable markers of autonomic activity, and inconsistent HRV measures). Here, we administered the Trier Social Stress Test (TSST), a validated stress-inducing task (Kirschbaum et al., 1993; Allen et al., 2017), to HTD patients and healthy controls, targeting a highly sensitive HRV parameter -i.e., the low frequency/high frequency (LF/HF) ratio- as well as electrophysiological and neuroimaging markers of heart-brain interactions.

Autonomic responses to induced mental stress are reliably indexed by HRV modulations (Taelman et al., 2009; Castaldo et al., 2015; Kim et al., 2018). Compared to other stress measures, HRV offers a relatively simple, non-invasive approach which has been increasingly used to assess autonomic function in various populations and paradigms (Thayer \& Sternberg, 2006; Vlcek et al., 2008; Chandola et al., 2010). Specifically, the LF/HF ratio outperforms single frequency-domain components in capturing the sympathovagal balance (Pagani et al., 1984; Task Force of the European Society of Cardiology and the North American Society of Pacing and Electrophysiology, 1996; Vlcek et al., 2008; Goldstein et al., 2011) during acute psychological stress (Xhyheri et al., 2012; Allen et al., 2014). In HTD patients, autonomic imbalance involves increased sympathetic and decreased parasympathetic activity at rest (Langewitz et al., 1994; Madsen et al., 2008; Thayer et al., 2010), alongside increased sympathetic reactivity (Kaushik et 
al., 2004; Wirtz et al., 2006) and lack of significant modulations of the LF/HF ratio (Garafova et al., 2014) during stress; all aligned with an impaired responsiveness to autonomic demands (Madsen et al., 2008). However, few studies have targeted the latter variable and most have favored sub-optimal mental stress inductors, such as the Stroop test (Garafova et al., 2014) or mental arithmetic paradigms (Ruediger et al., 2004), i.e., purely cognitive (non-socially-laden) tasks considered to induce small or inconsistent effects in isolation (Allen et al., 2014).

Furthermore, although studies that concurrently examined brain markers and peripheral measures in HTD have been encouraged (Jennings \& Zanstra, 2009), no previous report seems to have explored multidimensional brain correlates of stress in HTD. In particular, the use of a task indexed with structural and temporal brain measures may reveal different spatiotemporal mechanisms (Melloni et al., 2015; García-Cordero et al., 2016; Melloni et al., 2016; Ibáñez, 2018; Fittipaldi et al., 2020) underlying stress alterations, paving the way for new insights to inform the characterization and future treatments of this disease (Devor et al., 2013). To this end, we assessed ongoing modulations of the heartbeat evoked potential (HEP), an automatic cortical measure of interoceptive cardiac signals (Schandry \& Montoya, 1996; Pollatos et al., 2005) modulated by stress levels in healthy subjects (Schulz et al., 2013) and cardiac patients (Gray et al., 2007). Moreover, HEP modulations have been linked to HRV in normotensives (MacKinnon et al., 2013). Crucially, this neurophysiological marker is disrupted in HTD patients (Yoris et al., 2018), suggesting that sensing of autonomic visceral and vascular body signals (Craig, 2002; Critchley \& Harrison, 2013; Salamone et al., 2020) is associated with peripheral cardiovascular disruptions. This might reflect altered allostasis, that is, neural predictions of interoceptive signals to regulate the body's physiological system and support psychological phenomena including recognition of and reaction to psychosocial stress (Kleckner et al., 2017; Mocayar Marón et al., 2019). Also, from a neuroanatomical perspective, stress-induced HRV has been related to higher grey matter volume in fronto-temporo-insular regions in healthy subjects (Lane et al., 2009; Thayer et al., 2009; Thayer et al., 2012), whereas HTD patients show a disrupted association between brain volume and interoceptive performance (Yoris et al., 2018). In sum, HRV measures can be combined with HEP and neuroimaging to track the neurovisceral bases of stress in HTD.

We pursued this goal using the TSST, a test that reliably induces psychosocial stress in healthy and clinical populations (Heim et al., 2000; Dickerson \& Kemeny, 2004; Kudielka et al., 2007), including HTD patients (Wirtz et al., 2006). Specifically, the TSST achieves this by combining social and cognitive components that entail evaluative threat and outcome uncontrollability -two 
factors that surpass other stressors in eliciting stress responses such as cortisol levels (Dickerson \& Kemeny, 2004). Our multimodal approach encompasses measurements of a critical HRV feature (LF/HF ratio), neurophysiological (HEP), and neuroanatomical correlates (voxel-based morphometry [VBM]). Considering previous findings (Wirtz et al., 2006; Lane et al., 2009; Thayer et al., 2012; MacKinnon et al., 2013; Schulz et al., 2013; Garafova et al., 2014; Kleckner et al., 2017; Yoris et al., 2018; Mocayar Marón et al., 2019), we predicted that HTD patients would exhibit significantly increased and undifferentiated HRV (LF/HF ratio) during baseline and acute mental stress induced through the TSST. In this sense, higher HRV was expected to be accompanied by increased HEP modulations, associated to allostatic/interoceptive dysregulations. Finally, we expected that HTD patients would exhibit reduced associations between HRV and the volume of key fronto-temporo-insular regions. In brief, this multimodal study aims to shed novel light on multidimensional markers of health and mental stress.

\section{Materials and methods}

\section{Participants}

The study comprised 25 HTD patients and 27 healthy controls with no history of hypertension. Power estimation analysis confirmed the robustness of our sample size (Information S1). Subjects in the HTD group were chronic outpatients of the Metabolic and Arterial Hypertension Unit of the Favaloro Foundation Hospital, diagnosed following current revised criteria (Sánchez et al., 2003). Their blood pressure fell in Grade 1 (systolic blood pressure between 140-159 mmHg and/or diastolic blood pressure between 90-99 $\mathrm{mmHg}$ ), within the hypertension classification of the 2007 European Society of Hypertension (ESH) and the European Society of Cardiology (ESC) guidelines for the management of arterial hypertension (Mancia et al., 2007). Office blood pressure readings and ambulatory blood pressure monitoring confirmed the patients' condition (Table 1). All subjects in the HTD group were under antihypertensive medication (Table S1), but intake was suspended 48 hours before the study to prevent drug-related confounds (Yoris et al., 2018). None of the participants presented psychiatric symptoms, as assessed through extensive clinical interviews based on the WHO's ICD-10 diagnostic guidelines (WHO, 1992). Additionally, neither controls nor patients presented cognitive or emotional impairments (for details, see Cognitive and emotional assessment section, and Table 1), lacunar infarcts, or white (WM) or grey matter (GM) lesions (Figure S1). Both groups were matched for gender, age, education, and body 
mass index (Table 1). All participants signed an informed consent in accordance with the World Medical Association Declaration of Helsinki. The study was approved by the ethics committee of the Institute of Cognitive Neurology.

\section{Cognitive and emotional assessment}

All participants were evaluated with the INECO Frontal Screening (IFS) battery (Torralva et al., 2009), which is sensitive to executive dysfunction (Torralva et al., 2009; Gleichgerrcht et al., 2011). This test includes eight subtests: (1) motor programming (Luria series, “'fist, edge, palm'); (2) conflicting instructions (subjects must hit the table once when the administrator hits it twice, or twice when the administrator hits it only once); (3) motor inhibitory control; (4) numerical working memory (backward digit span); (5) verbal working memory (months backwards); (6) spatial working memory (modified Corsi tapping test); (7) abstraction capacity (inferring the meaning of proverbs); and (8) verbal inhibitory control (modified Hayling test). The maximum possible score on the IFS is 30 points. Furthermore, we used the Beck Depression Inventory (BDIII) (Beck et al., 1996), a 21-item scale for the assessing of emotional, behavioral, and somatic symptoms of depression. Higher BDI-II scores indicate more severe depression symptoms. Between-group comparisons assessed through one-way ANOVA indicated non-significant differences between controls and HTD patients (Table 1).

\section{Trier Social Stress Test}

Responses to stress were assessed with the TSST (Kirschbaum et al., 1993; Allen et al., 2017), a gold-standard test to induce acute psychosocial stress, as indexed through key biomarkers (Dickerson \& Kemeny, 2004; Allen et al., 2014). In light of our hypothesis, we focused on two periods of the test: the baseline period (used to determine a basal state) and the stress period (which involves three stressful tasks: speech preparation, speech, and mental arithmetic). First, in the baseline period (lasting 5 minutes), participants were asked to remain still, keep their eyes open, and avoid thinking about anything in particular. Then, subjects were given 5 minutes to prepare a speech and convince an evaluator that they were perfect candidates for a job (Speech preparation). Immediately after, participants delivered their monologue facing an unfamiliar evaluator trained to withhold any type of social engagement or positive feedback (Speech). Finally, participants completed a 5-minute serial-subtraction task (Mental arithmetic), assessed by the same evaluator. ECG and EEG signals were recorded during the whole test to assess HRV and 
heart rate (see ECG data preprocessing and analysis section for details), as well as HEP modulations (see EEG data preprocessing and analysis section for details), respectively.

\section{State-anxiety assessment during TSST}

Exposure to stress generally leads to subjectively negative experiences (Allen et al., 2014), and the TSST, in particular, increases anxiety (Kirschbaum et al., 1993; Rohrmann et al., 1999; Rimmele et al., 2009). To confirm that participants, especially the HTD sample, found the TSST stressful, we compared state anxiety using the state scale of the State-Trait Anxiety Inventory (STAI) (Speilberger \& Vagg, 1984) before baseline and immediately after stress period. Twotailed paired $t$-tests showed increased anxiety across groups immediately after the stress period, evidencing that the task was actually stressful (for details, see Table 2).

\section{ECG data preprocessing and analysis}

\section{Preprocessing of heart rate data}

We measured HRV to estimate the influence of cardiodynamic differences between groups during the TSST (Castaldo et al., 2015). We used the Kubios HRV program (Tarvainen et al., 2014) and imported the beat-to-beat RR interval data from the ECG (on the MATLAB platform [MATLAB R2017b, The MathWorks, Natick, MA, USA]). This software analyzes HRV in both time and frequency domains. Given the short timespans, we applied an autoregressive algorithm to analyze the power spectrum (Task Force of the European Society of Cardiology and the North American Society of Pacing and Electrophysiology, 1996). This algorithm generates a power spectral analysis with different frequency bands: high frequency (HF: 0.15 to $0.40 \mathrm{~Hz}$ ), low frequency (LF: 0.04 to $0.15 \mathrm{~Hz}$ ), and very low frequency (VLF: 0.00 to $0.04 \mathrm{~Hz}$ ). The LF/HF ratio has been suggested as a measure of sympathovagal balance (Eckberg, 1997; Taelman et al., 2009). We calculated these frequency components in normalized units, which represent the relative value of each power component in proportion to the total power minus the VLF component (Pagani et al., 1986; Malliani, 1991). Moreover, The Kubios HRV also yields a mean heart rate measure for each period.

\section{HRV analysis}

HRV was analyzed based on normalized units from the LF/HF ratio. To remove data unrelated to psychophysiological process and enhance the power, values above 2 SDs LF/HF ratio were 
excluded (Zimmerman, 1994; Osborne \& Overbay, 2004; de la Fuente et al., 2019). The mean HRV (LF/HF) modulation during the TSST periods (baseline and stress) was compared between groups via a one-way ANOVA. Effect sizes were reported with partial eta squared $\left(\eta p^{2}\right)$. Betweengroup comparisons were performed on SPSS software (version 22.0, IBM Corp, Armonk, NY, USA).

\section{Heart rate analysis}

Acute stress raises heart rate, as confirmed in previous TSST studies (Buske-Kirschbaum et al., 2002; Rimmele et al., 2007; Yamakawa et al., 2009). We assessed heart rate differences between periods for each group via two-tailed paired $t$-tests. Both groups presented a significant increase in heart rate between baseline and stress periods (see Table 3), confirming that the stress period of the TSST was actually stressful.

\section{EEG data preprocessing and analysis}

\section{EEG signal preprocessing}

Data was obtained with a Biosemi Active-two 128-channel system at $1024 \mathrm{~Hz}$. Preprocessing and analysis were performed using EEGLAB functions (EEGLAB 14.1.1b, University of San Diego, San Diego, CA, USA), running under MATLAB. EEG data was resampled offline at 256 $\mathrm{Hz}$ and band-pass filtered during recording $(0.1$ to $100 \mathrm{~Hz})$ and off-line $(0.3$ to $50 \mathrm{~Hz})$ to remove undesired frequency components (Yoris et al., 2018). The reference was set by default to link mastoids. EEG data were segmented between -200 to $700 \mathrm{~ms}$ of the ECG R-wave peak. Following a widely reported EEG approach (Couto et al., 2014; Couto et al., 2015a; García-Cordero et al., 2016; Garcia-Cordero et al., 2017; Yoris et al., 2017; Salamone et al., 2018; Yoris et al., 2018; Fittipaldi et al., 2020), segments with cardiac-field artifacts and eye movement contamination (including slow and fast blinks, and vertical and horizontal eye movements) were rejected via independent component analysis (ICA). In this analysis, spatial filters are derived by producing the set of maximally temporally independent signals in the EEG data (Delorme et al., 2007). The components of the data that resemble electromyogenic artifacts (broad bandwidth, peripheral distribution) were selected through visual inspection by a well-trained expert and projected out of the channel, leaving clean data only. To avoid possible biases related to the rejection criteria, the same expert was in charge of the whole visual inspection procedure, a standard for artifact 
rejection in EEG research (Muthukumaraswamy, 2013). The epochs were baseline-corrected (baseline: -200 ms to $0 \mathrm{~ms}$ ) (Szczepanski et al., 2014). Finally, noisy epochs were also rejected following a visual inspection procedure (Yoris et al., 2018). No significant differences were found in the number of remaining epochs between groups in each period (Information S3).

\section{HEP analysis}

The HEP was obtained by sampling EEG epochs time-locked to the R-wave. HEP modulations between TSST baseline and TSST stress were analyzed through a point-by-point Monte Carlo permutation test (5000 permutations, $P<0.05$ ) (Manly, 2006), as done previously with this ERP (Couto et al., 2015b; García-Cordero et al., 2016; Yoris et al., 2018; Salamone et al., 2020) and other components (Ibanez et al., 2012; Ibáñez et al., 2013; Amoruso et al., 2014; Melloni et al., 2016). This method offers a solution to the multiple comparisons problem and does not depend on multiple comparison corrections or Gaussian distribution assumptions (Nichols \& Holmes, 2002). We analyzed the HEP signal only after the 200-ms mark to avoid the influence of cardiac field artifacts (Kern et al., 2013). HEP analyses were based on an extended frontal region of interest (ROI) (40 frontal electrodes: Biosemi C1 C2 C3 C4 C5 C6 C7 C8 C9 C10 C11 C12 C13 C14 C15 C16 C17 C18 C19 C20 C21 C22 C23 C24 C25 C26 C27 C28 C29 C30 C31 C32 D1 D2 D3 D4 D5 D6 D7 D8) (Figure 1b) (Pollatos \& Schandry, 2004; Gray et al., 2007; Yoris et al., 2018). To examine the topographic distribution within the extended frontal ROI, we also assessed three separate sub-ROIs within the above set of electrodes, namely: a right frontal (Biosemi C1 C2 C3 C4 C5 C6 C7 C8 C9 C10 C11 C12 C13), a left frontal (Biosemi C24 C25 C26 C30 C31 C32 D1 D2 D3 D4 D5 D6 D7), and a central frontal (Biosemi C14 C15 C16 C17 C18 C19 C20 C21 C22 C23 C27 C28 C29) ROI (Figure S2).

\section{Associations between HEP and HRV}

Pearson's correlation analyses $(P<0.05)$ were used to examine a possible association between HEP modulation and HRV during the TSST, in both groups. This was done by calculating the mean average of the significant window of the HEP modulation during each TSST period (baseline and stress).

\section{MRI Measures}

\section{MRI acquisition and preprocessing}


MRI acquisition and preprocessing steps are reported as recommended by the Organization for Human Brain Mapping (Nichols et al., 2017; Poldrack et al., 2017). Eight subjects from the original sample were excluded due to absence of imaging data, artifacts or acquisition problems. The final MRI sample consisted of 23 controls and 21 HTD patients demographically matched (see Table S2). Using a 1.5-T Phillips Intera scanner with a standard head coil (8 channels), we acquired whole-brain T1-weighted anatomical 3D spin echo volumes, parallel to the plane connecting the anterior and posterior commissures, with the following parameters: repetition time $(\mathrm{TR})=7489 \mathrm{~ms}$; echo time $(\mathrm{TE})=3420 \mathrm{~ms}$; flip angle $=8^{\circ}$; 196 slices, matrix dimension $=256 \mathrm{x}$ 240; voxel size $=1 \times 1 \times 1 \mathrm{~mm} 3$; sequence duration $=7 \mathrm{~min}$.

The resulting images were preprocessed on the DARTEL Toolbox, following validated procedures (Ashburner \& Friston, 2000; Yoris et al., 2018), and analyzed on Statistical Parametric Mapping software (SPM12, Wellcome Department of Cognitive Neurology, University of London, London, UK, available in http://www.fil.ion.ucl.ac.uk/spm/software/spm12/) running on MATLAB. T1-weighted images in native space were first segmented using the default parameters of SPM12 (bias regularization was set to 0.001, and bias FWHM was set to 60-mm cut-off) into WM, GM, and cerebrospinal fluid (used to estimate the total intracranial volume). Then we ran the "DARTEL (create template)" module using the GM and WM segmented images - with the default parameters - to create a template from the complete data set (to increase the accuracy of intersubject alignment) (Ashburner, 2007). Then, we used the "Normalise to MNI Space" module from DARTEL Tools to affine register the last template from the previous step into the MNI Space. This transformation was then applied to all the individual GM segmented scans to also be brought into standard space. Subsequently, all images were modulated to correct volume changes by Jacobian determinants, and avoid a bias in the intensity of an area due to its expansion during warping. Finally, data were smoothed using an $8 \mathrm{~mm}$ full-width-at-half-maximum isotropic Gaussian kernel to accommodate for inter-subject differences in anatomy. The size of the kernel was selected based on previous recommendations (Good et al., 2001).

\section{Association between HRV during TSST and neuroimaging measures}

Regression analyses were performed to account for the relation between HRV in TSST periods (baseline and stress) and GM, using the outputs from VBM preprocessing. Given that HRV has been related to a widely distributed set of fronto-temporo-insular regions (Lane et al., 2009; Thayer et al., 2012), we applied a specific mask comprising these areas (anterior cingulate cortex, 
orbitofrontal cortex, gyrus rectus, inferior frontal gyrus, anterior frontal middle gyrus, amygdala, basal ganglia [caudate nucleus, putamen, pallidum], insula, hippocampus, and parahippocampus) and performed a voxel-wise analysis inside them (Sedeno et al., 2016; de la Fuente et al., 2019; Bachli et al., 2020). This mask was developed based on the regions of the Automated Anatomical Labeling (AAL) Atlas (Tzourio-Mazoyer et al., 2002). Total intracranial volume was used as a covariate to discard the influence of brain-size differences. However, differences in overall brain volume between groups were assessed through one-way ANOVA. No significant differences were found $\left(F_{1,42}=0.00, P=0.992, \eta p^{2}<0.01\right)$ between patients $(M=1386.73, S D=128.83)$ and controls $(M=1386.35, S D=122.66)$. Statistical significance was set at $P<0.001$ uncorrected, extent threshold $=30$ voxels (Steeb et al., 2018; de la Fuente et al., 2019). A power estimation analysis confirmed the robustness of our results (Information S2).

\section{Results}

\section{HRV during the TSST}

Between-group comparisons of HRV during the TSST via one-way ANOVA revealed significant differences for the baseline period $\left(F_{1,46}=10.54, P=0.00, \eta \mathrm{p}^{2}=0.18\right)$, such that patients $(M=4.30, S D=3.97)$ showed higher $\mathrm{LF} / \mathrm{HF}$ ratio than controls $(M=1.58, S D=1.27)$.

No significant differences were found in the stress period $\left(F_{1,40}=0.37 P=0.54, \eta \mathrm{p}^{2}=0.009\right)$ between patients $(M=3.34, S D=1.87)$ and controls $(M=3.66, S D=1.36)$ (Figure 1a).

Also, to test for potential group differences in the transition between periods (specifically, the groups' adjustment to the stress period), we subtracted baseline HRV from stress HRV for each sample, thus controlling for baseline fluctuations and adjusting for normal intrasubject variability. A one-way ANOVA between both subtractions showed significant differences $\left(F_{1,38}=6.37 P=\right.$ $\left.0.01, \eta p^{2}=0.14\right)$, with lower values for patients $(M=-0.60, S D=2.99)$ than controls $(M=1.63$, $S D=1.78)$ (Figure 1a).

The same results were obtained when the sample was analyzed without excluding outliers. Between-group comparisons of HRV during the TSST revealed significant differences for the baseline period $\left(F_{1,50}=5.39, P=0.02, \eta p^{2}=0.09\right)$, such that patients $(M=4.82, S D=5.20)$ showed higher $\mathrm{LF} / \mathrm{HF}$ ratio than controls $(M=2.20, S D=2.60)$. In the stress period, no significant differences were found $\left(F_{1,50}=0.04, P=0.82, \eta \mathrm{p}^{2}=0.001\right)$ between patients $(M=$ $3.99, S D=2.39)$ and controls $(M=4.18, S D=3.66)$. Finally, when comparing the groups' 
transition between periods, a one-way ANOVA between subtractions of HRV conditions showed significant differences $\left(F_{1,50}=4.29, P=0.04, \eta p^{2}=0.07\right)$, with lower values for patients $(M=$ $0.83, S D=5.55)$ than controls $(M=1.97, S D=4.18)$.

\section{HEP modulation during the TSST and association with HRV}

During the baseline period, significantly greater HEP modulations were observed in the patients compared to controls over the frontal ROI between 566 and $589 \mathrm{~ms}(P<0.05)$ (Figure 1b). Conversely, the stress period yielded no significant differences between groups. These results were replicated $(P<0.05)$ over the right frontal [460 to $480 \mathrm{~ms} ; 562$ to $597 \mathrm{~ms}$ ] and left frontal [570 to $593 \mathrm{~ms}$ ] sites when each sub-ROI was separately assessed (Figure S2).

In the baseline period, a significant negative association between the HEP and HRV was observed in controls $(r=-0.49, P=0.02)$ and patients $(r=-0.51, P=0.05)$. Conversely, the stress period yielded no such correlation in either group (Controls: $r=-0.16, P=0.51$; patients: $r=$ $0.28, P=0.46)$ (Figure 1c).

\section{Association between HRV and GM volume}

HRV during baseline was associated with the GM volume of the right middle orbital frontal cortex and left insula in controls, and with the left middle frontal cortex in patients. In contrast, during the stress period, controls presented a significant positive association between HRV and GM volume of right inferior and superior frontal cortices as well as the right putamen. No significant correlations were found in patients in the stress period (Figure 1d and Table 4).

\section{Discussion}

Our results evidence stress-related HRV dysregulation in HTD associated with electrophysiological and neuroimaging markers. Relative to controls, patients exhibited increased LF/HF ratio and greater HEP modulations during baseline, as well as reduced changes between baseline and stress periods, and lack of significant stress-related HRV associations with GM volume of putative frontrostriatal regions. This novel multidimensional evidence suggests that HTD patients present core markers of increased stress in non-stressful conditions and impaired neurovisceral responsiveness to acute stress. Altogether, our findings add to a promising theoretical and clinical agenda. 
First, HTD patients exhibited increased LF/HF ratio during baseline, which has been associated to basal sympathetic hyperactivity and parasympathetic withdrawal (Wirtz et al., 2006; Madsen et al., 2008; Xhyheri et al., 2012; Garafova et al., 2014). This pattern is indicative of basal autonomic imbalance. More particularly, since these same modulations are core signatures of mental stress in healthy subjects (Kim et al., 2018), our results may indirectly represent markers of basal stress in HTD, possibly emerged from a constant overreaction to daily life stress (Kaushik et $a l ., 2004)$. In this line, greater frontal HEP modulations found in patients during baseline, could be interpreted as an interoceptive hypervigilance driven by a stress overload. Indeed, cortisol levels have been related to HEP amplitudes (Schulz et al., 2013). Also, the absence of significant associations between HRV and GM volume of key brain regions subserving cardiac activity strengthens the view that neurovisceral regulations are disrupted in HTD compared to controls. Indeed, controls' HRV was related with regions' GM volume underlying adaptive behavior, emotion regulation, and autonomic regulation (Lane et al., 2009; Thayer et al., 2012), namely: the right middle orbital frontal cortex (Thayer et al., 2009) and left insula (Oppenheimer et al., 1996; Ibanez et al., 2010; Wei et al., 2018; Ibáñez, 2019). The association between HRV and HEP in controls (MacKinnon et al., 2013; Shaffer et al., 2014) was replicated during baseline. However, the patients' reduced association (only marginally significant) might suggest suboptimal neurovisceral interactions. Briefly, these results indicate that, even in the absence of explicit stressors, HTD patients present multimodal signatures of altered stress-related mechanisms, suggesting a basal stress overload effect.

The above interpretation is consistent with results from the stress period. Although no HRV differences were found between groups during stress, the significantly smaller between-periods changes in LF/HF ratio observed in HTD reflects the absence of HRV adjustment to acute mental stress. The lack of endogenous variability in neurally mediated peripheral systems reinforces the view of impaired autonomic responsiveness (Madsen et al., 2008; Thayer et al., 2010; Shaffer et al., 2014). Indeed, this pattern could be explained by the exacerbated modulations of these markers during baseline. However, other studies did report increased sympathetic activity during stress in HTD (Kaushik et al., 2004; Nyklicek et al., 2005; Wirtz et al., 2006). While that may seem at odds with our findings, most of these studies used autonomic measures that differ from the present HRV index. Also, studies that use the LF/HF ratio tracked markedly younger samples (characterized by increased sympathetic activity) and suboptimal stress-inducing methods (Ruediger et al., 2004; Garafova et al., 2014). Moreover, during the stress period, the lack of 
significant GM associations with HRV modulations in the HTD group compared to controls and altered HEP modulations (Gray et al., 2007) supports the hypothesis of homeostatic disruptions and neurovisceral interaction disturbances in acute stress. Indeed, these dynamics are markedly different in healthy subjects. As corroborated in our study, normotensives show greater betweenperiods changes in LF/HF ratio, as well as right-sided frontrostriatal GM volume correlates during task-evoked ANS regulation (Lane et al., 2009; Thayer et al., 2009; Wei et al., 2018). The absence of these markers of normal autonomic reactivity to stress (Delaney \& Brodie, 2000; Castaldo et al., 2015; Kim et al., 2018) and healthy ANS balance (Thayer et al., 2010) in HTD could explain the loss of significant differences between groups in HRV modulation and HEP amplitude during stress. Altogether, these findings further point to defective multidimensional responsiveness to acute mental stress in HTD patients.

Our findings carry theoretical and clinical implications. Basal autonomic imbalance and the lack of adjustment to acute stress in HTD could be explained through the Neurovisceral Integration Across a Continuum of Time (NIACT) model (Kemp et al., 2017), a novel temporal framework which characterizes HRV as a link between psychological moments and mortality risk. Specifically, it conceptualizes how everyday moments both affect and are affected by the HRV in ways that have long-term effects on mortality risk, since it indexes the vagus nerve -which plays a critical role over allostatic systems. Consequently, basal HRV may index everyday psychophysiological resources, providing the best indication of wellbeing and future health. Conversely, task-driven activity, including psychosocial stress, would reflect autonomic responsiveness (Kemp et al., 2017). In this line, increased basal LF/HF ratio in HTD suggests peripheral cardiovascular disruptions triggering allostatic load (McEwen, 1998). Our results also extend the NIACT model onto neurocognitive dimensions, by showing the HEP and GM density in fronto-temporo-insular areas constitute key markers of neurovisceral interaction. For instance, the differential HEP modulation observed between groups at baseline may reflect the abovementioned allostatic overload in HTD. This aligns with an embodied perspective suggesting that peripheral cardiovascular impairments, previously associated to interoceptive deficits (Yoris et al., 2018), may disrupt allostatic-interoceptive dynamics, preventing successful allostasis. More specifically, the greater frontal HEP modulations found in patients during baseline could be interpreted as interoceptive hypervigilance driven by stress-related allostatic overload. Moreover, the pathophysiological implications of this state (Karatsoreos \& McEwen, 2011) might explain the development and maintenance of hypertension (Liu et al., 2017b; Mocayar Marón et al., 2019) 
and its detrimental impact on patients' health (Ruediger et al., 2004; Thayer et al., 2010). Further developments along these lines can lead to more comprehensive modelling of stress in healthy and pathological populations.

From a clinical perspective, our results highlight basal HRV monitoring as an objectively cardio-cognitive marker in risk populations. Similarly, multilevel neurovisceral indicators could afford objective assessments of the patients' ability to adaptively react to acute psychosocial stressors, complementing standard self-report evaluations (Kim et al., 2018). In line with previous works (García-Cordero et al., 2016; Salamone et al., 2018; Yoris et al., 2018; Fittipaldi et al., 2020), these multimodal insights underscore the relevance of neurocognitive data for the development of applied innovations in the diagnosis, prognosis, and treatment of conditions with autonomic imbalance and increased morbidity (Thayer et al., 2010; Castaldo et al., 2015; Allen et al., 2017; Kemp et al., 2017).

\section{Limitations and avenues for further research}

The present work features some limitations and opens a new agenda for further research. First, it is based on a modest sample size. However, it is adequate according to power estimation analysis (Information S1 and S2) and similar to other works with convergent results (Wirtz et al., 2006; Garafova et al., 2014). Moreover, the consistency of our results across cardiodynamic, electrophysiological, and neuroimaging dimensions, with moderate to large effect sizes, further attests to their robustness. Still, non-significant results may vary with larger samples, calling for further replication. Second, our findings may have been partially influenced by the patients' medication. However, following previous reports (Yoris et al., 2018), we suspended intake 48 hours before testing to minimize its potential impact. Still, valuable information could be obtained in future works by comparing basal and acute stress levels in treated and untreated patients. Another possible limitation is the restricted range of stress parameters. Although HRV and HEP are valid proxies to assess acute mental stress, future studies should include other parameters, like salivary cortisol (Kirschbaum \& Hellhammer, 1994; Kudielka et al., 2007) hypothalamuspituitary-adrenal axis activity, sympathetic-adrenal-medullary activation or immune system activity measurements (Nyklicek et al., 2005; Wirtz et al., 2006). Moreover, the sensitivity of the $\mathrm{LF} / \mathrm{HF}$ ratio to sympatho-vagal balance is debated, especially in short-term resting paced breathing (at $0.1 \mathrm{~Hz}$ ) recordings, where LF power may be almost entirely vagally mediated (Billman, 2013; Shaffer et al., 2014). Nonetheless, in our work, we assumed that the LF component mainly 
reflected sympathetic activity, since the TSST involved a baseline period assessed at normal breathing and a psychosocial stress period that has been widely validated to produce sympathetic activation (Allen et al., 2014; Allen et al., 2017). We encourage future studies to take this issue into consideration. Finally, between-group HEP differences in the present study emerged in a short time span. However, this ERP may manifest in windows of variable extension (Gray et al., 2007; Schulz et al., 2015; Salamone et al., 2018; Salamone et al., 2020). Future studies could illuminate which subject- or task-related factors determine the duration of (stress-sensitive) HEP effects.

\section{Conclusions}

Our study revealed an autonomic imbalance in HTD patients, reflected in a potential basal stress overload effect and a lack of regulation to acute psychosocial stress, accompanied by neurophysiological and neuroanatomical alterations. Further work along these lines could better elucidate the multidimensional signatures of stress in HTD patients and normotensives, yielding new insights into heart-brain interactions.

\section{Acknowledgements}

We are grateful to the Metabolic and Arterial Hypertension Unit of the Favaloro Foundation Hospital for their collaboration.

\section{Financial support}

This work was supported by CONICET; FONCYT-PICT [2017-1818, 2017-1820]; CONICYT/FONDECYT Regular [grant number 1170010]; FONDAP [grant number 15150012]; GBHI ALZ UK-20-639295; and the Multi-Partner Consortium to Expand Dementia Research in Latin America (ReDLat), funded by the National Institutes of Aging of the National Institutes of Health under award number R01AG057234, an Alzheimer's Association grant (SG-20-725707), the Rainwater Foundation, and the Global Brain Health Institute. The content is solely the responsibility of the authors and does not represent the official views of these institutions.

\section{Conflict of Interest Statement}

The authors have no conflicts of interest to declare.

\section{Author Contributions}


Study Design: AY, AI, LS.

Data collection: AY, SA, MM, FA.

Data processing and analysis: AL and LS.

Wrote manuscript: AL, AI, and AG.

All authors have participated sufficiently in the work and approve the final version of the manuscript for submission.

\section{Data Accessibility Statement}

Experimental data is available online on the Open Science Framework at: https://osf.io/ybgn5.

\section{Abbreviations}

ANS: autonomic nervous system

GM: grey matter

HEP: heart-evoked potential

HRV: heart rate variability

HTD: hypertensive disease

$\mathrm{LF} / \mathrm{HF}$ : low frequency/high frequency

NIACT: Neurovisceral Integration Across a Continuum of Time model

ROI: region of interest

SPM12: Statistical Parametric Mapping software v.12

TSST: Trier Social Stress Test

VBM: voxel-based morphometry

WM: white matter

\section{References}

Allen, A.P., Kennedy, P.J., Cryan, J.F., Dinan, T.G. \& Clarke, G. (2014) Biological and psychological markers of stress in humans: focus on the Trier Social Stress Test. Neurosci. Biobehav. Rev, 38, 94-124.

Allen, A.P., Kennedy, P.J., Dockray, S., Cryan, J.F., Dinan, T.G. \& Clarke, G. (2017) The Trier Social Stress Test: Principles and practice. Neurobiol. Stress., 6, 113-126. 
Amoruso, L., Sedeño, L., Huepe, D., Tomio, A., Kamienkowski, J., Hurtado, E., Cardona, J.F., González, M.Á.Á., Rieznik, A. \& Sigman, M. (2014) Time to tango: expertise and contextual anticipation during action observation. NeuroImage, 98, 366-385.

Ashburner, J. (2007) A fast diffeomorphic image registration algorithm. NeuroImage, 38, 95-113.

Ashburner, J. \& Friston, K.J. (2000) Voxel-based morphometry - the methods. NeuroImage, 11, 805-821.

Bachli, M.B., Sedeño, L., Ochab, J.K., Piguet, O., Kumfor, F., Reyes, P., Torralva, T., Roca, M., Cardona, J.F. \& Campo, C.G. (2020) Evaluating the reliability of neurocognitive biomarkers of neurodegenerative diseases across countries: A machine learning approach. NeuroImage, 208, 116456.

Beck, A.T., Steer, R.A. \& Brown, G.K. (1996) Beck depression inventory-II. San Antonio, 78, 490-498.

Billman, G.E. (2013) The LF/HF ratio does not accurately measure cardiac sympatho-vagal balance. Front. Physiol., 4, 26.

Buske-Kirschbaum, A., Geiben, A., Höllig, H., Morschhäuser, E. \& Hellhammer, D. (2002) Altered responsiveness of the hypothalamus-pituitary-adrenal axis and the sympathetic adrenomedullary system to stress in patients with atopic dermatitis. J. Clin. Endocrinol. Metab., 87, 4245-4251.

Castaldo, R., Melillo, P., Bracale, U., Caserta, M., Traissi, M. \& Pecchia, L. (2015) Acute mental stress assessment via short term HRV analysis in healthy adults: A systematic review with meta-analysis. Biomed. Signal Process. Control, 18, 370-377. 
Cohen, S., Janicki-Deverts, D. \& Miller, G.E. (2007) Psychological stress and disease. Jama, 298, 1685-1687.

Couto, B., Adolfi, F., Velasquez, M., Mesow, M., Feinstein, J., Canales-Johnson, A., Mikulan, E., Martinez-Pernia, D., Bekinschtein, T., Sigman, M., Manes, F. \& Ibanez, A. (2015a) Heart evoked potential triggers brain responses to natural affective scenes: A preliminary study. Autonomic neuroscience : basic \& clinical, 193, 132-137.

Couto, B., Adolfi, F., Velasquez, M., Mesow, M., Feinstein, J., Canales-Johnson, A., Mikulan, E., Martinez-Pernia, D., Bekinschtein, T., Sigman, M., Manes, F. \& Ibanez, A. (2015b) Heart evoked potential triggers brain responses to natural affective scenes: A preliminary study. Auton. Neurosci., 193, 132-137.

Couto, B., Salles, A., Sedeno, L., Peradejordi, M., Barttfeld, P., Canales-Johnson, A., Dos Santos, Y.V., Huepe, D., Bekinschtein, T., Sigman, M., Favaloro, R., Manes, F. \& Ibanez, A. (2014) The man who feels two hearts: the different pathways of interoception. Social cognitive and affective neuroscience, 9, 1253-1260.

Craig, A.D. (2002) How do you feel? Interoception: the sense of the physiological condition of the body. Nat. Rev Neurosci., 3, 655-666.

Critchley, H.D. \& Harrison, N.A. (2013) Visceral influences on brain and behavior. Neuron, 77, 624-638.

Chandola, T., Heraclides, A. \& Kumari, M. (2010) Psychophysiological biomarkers of workplace stressors. Neurosci. Biobehav. Rev., 35, 51-57. 
de la Fuente, A., Sedeño, L., Schurmann, S., Ellmann, C., Sonzogni, S., Belluscio, L., GarcíaCordero, I., Castagnaro, E., Boano, M. \& Cetkovich, M. (2019) Multimodal neurocognitive markers of interoceptive tuning in smoked cocaine. Neuropsychopharmacology, 44, 1425-1434.

Delaney, J. \& Brodie, D. (2000) Effects of short-term psychological stress on the time and frequency domains of heart-rate variability. Percept. Mot. Ski., 91, 515-524.

Delorme, A., Sejnowski, T. \& Makeig, S. (2007) Enhanced detection of artifacts in EEG data using higher-order statistics and independent component analysis. NeuroImage, 34, 14431449.

Devor, A., Bandettini, P.A., Boas, D.A., Bower, J.M., Buxton, R.B., Cohen, L.B., Dale, A.M., Einevoll, G.T., Fox, P.T. \& Franceschini, M.A. (2013) The challenge of connecting the dots in the BRAIN. Neuron, 80, 270-274.

Dickerson, S.S. \& Kemeny, M.E. (2004) Acute stressors and cortisol responses: a theoretical integration and synthesis of laboratory research. Psychol. Bull., 130, 355.

Eckberg, D.L. (1997) Sympathovagal balance: a critical appraisal. Circulation, 96, 3224-3232.

Fittipaldi, S., Abrevaya, S., de la Fuente, A., Pascariello, G.O., Hesse, E., Birba, A., Salamone, P., Hildebrandt, M., Martí, S.A. \& Pautassi, R. (2020) A multidimensional and multi-feature framework for cardiac interoception. NeuroImage, 116677.

Garafova, A., Penesova, A., Cizmarova, E., Marko, A., Vlcek, M. \& Jezova, D. (2014) Cardiovascular and sympathetic responses to a mental stress task in young patients with hypertension and/or obesity. Physiol. Res., 63, S459. 
Garcia-Cordero, I., Esteves, S., Mikulan, E.P., Hesse, E., Baglivo, F.H., Silva, W., Garcia, M.D.C., Vaucheret, E., Ciraolo, C., Garcia, H.S., Adolfi, F., Pietto, M., Herrera, E., Legaz, A., Manes, F., Garcia, A.M., Sigman, M., Bekinschtein, T.A., Ibanez, A. \& Sedeno, L. (2017) Attention, in and Out: Scalp-Level and Intracranial EEG Correlates of Interoception and Exteroception. Frontiers in neuroscience, 11, 411.

García-Cordero, I., Sedeño, L., De la Fuente, L., Slachevsky, A., Forno, G., Klein, F., Lillo, P., Ferrari, J., Rodriguez, C., Bustin, J., Torralva, T., Baez, S., Yoris, A., Esteves, S., Melloni, M., Salamone, P., Huepe, D., Manes, F., A., G.M. \& Ibañez, A. (2016) Feeling, learning from and being aware of inner states: interoceptive dimensions in neurodegeneration and stroke. Philos. Trans. R. Soc. B., 371, 20160006.

Gianaros, P.J. \& Sheu, L.K. (2009) A review of neuroimaging studies of stressor-evoked blood pressure reactivity: emerging evidence for a brain-body pathway to coronary heart disease risk. NeuroImage, 47, 922-936.

Gleichgerrcht, E., Roca, M., Manes, F. \& Torralva, T. (2011) Comparing the clinical usefulness of the Institute of Cognitive Neurology (INECO) Frontal Screening (IFS) and the Frontal Assessment Battery (FAB) in frontotemporal dementia. J. Clin. Exp. Neuropsychol., 33, 997-1004.

Goldstein, D.S., Bentho, O., Park, M.Y. \& Sharabi, Y. (2011) Low-frequency power of heart rate variability is not a measure of cardiac sympathetic tone but may be a measure of modulation of cardiac autonomic outflows by baroreflexes. Exp. physiol., 96, 1255-1261.

Good, C.D., Johnsrude, I.S., Ashburner, J., Henson, R.N., Friston, K.J. \& Frackowiak, R.S. (2001) A voxel-based morphometric study of ageing in 465 normal adult human brains. NeuroImage, 14, 21-36. 
Gray, M.A., Taggart, P., Sutton, P.M., Groves, D., Holdright, D.R., Bradbury, D., Brull, D. \& Critchley, H.D. (2007) A cortical potential reflecting cardiac function. Proc. Natl. Acad. Sci. U. S. A. , 104, 6818-6823.

Heim, C., Ehlert, U. \& Hellhammer, D.H. (2000) The potential role of hypocortisolism in the pathophysiology of stress-related bodily disorders. Psychoneuroendocrinology, 25, 1-35.

Ibanez, A., Gleichgerrcht, E. \& Manes, F. (2010) Clinical effects of insular damage in humans. Brain structure \& function, 214, 397-410.

Ibanez, A., Melloni, M., Huepe, D., Helgiu, E., Rivera-Rei, A., Canales-Johnson, A., Baker, P. \& Moya, A. (2012) What event-related potentials (ERPs) bring to social neuroscience? Soc Neurosci, 7, 632-649.

Ibáñez, A. (2018) Brain oscillations, inhibition and social inappropriateness in frontotemporal degeneration. Brain : a journal of neurology, 141, e73.

Ibáñez, A. (2019) Insular networks and intercognition in the wild. Cortex; a journal devoted to the study of the nervous system and behavior, 115, 341-344.

Ibáñez, A., Cardona, J.F., Dos Santos, Y.V., Blenkmann, A., Aravena, P., Roca, M., Hurtado, E., Nerguizian, M., Amoruso, L. \& Gómez-Arévalo, G. (2013) Motor-language coupling: direct evidence from early Parkinson's disease and intracranial cortical recordings. Cortex; a journal devoted to the study of the nervous system and behavior, 49, 968-984.

Jennings, J.R. \& Zanstra, Y. (2009) Is the brain the essential in hypertension? NeuroImage, 47, 914-921. 
Karatsoreos, I.N. \& McEwen, B.S. (2011) Psychobiological allostasis: resistance, resilience and vulnerability. Trends Cogn. Sci., 15, 576-584.

Kaushik, R.M., Mahajan, S.K., Rajesh, V. \& Kaushik, R. (2004) Stress profile in essential hypertension. Hypertens. Res., 27, 619-624.

Kemp, A.H., Koenig, J. \& Thayer, J.F. (2017) From psychological moments to mortality: a multidisciplinary synthesis on heart rate variability spanning the continuum of time. Neurosci. Biobehav. Rev., 83, 547-567.

Kern, M., Aertsen, A., Schulze-Bonhage, A. \& Ball, T. (2013) Heart cycle-related effects on event-related potentials, spectral power changes, and connectivity patterns in the human ECoG. NeuroImage, 81, 178-190.

Kim, H.G., Cheon, E.J., Bai, D.S., Lee, Y.H. \& Koo, B.H. (2018) Stress and heart rate variability: A meta-analysis and review of the literature. Psychiatry Investig., 15, 235.

Kirschbaum, C. \& Hellhammer, D.H. (1994) Salivary cortisol in psychoneuroendocrine research: recent developments and. Psychoneuroendocrinology, 19, 313-333.

Kirschbaum, C., Pirke, K.M. \& Hellhammer, D.H. (1993) The 'Trier Social Stress Test'-a tool for investigating psychobiological stress responses in a laboratory setting. Neuropsychobiology, 28, 76-81.

Kleckner, I.R., Zhang, J., Touroutoglou, A., Chanes, L., Xia, C., Simmons, W.K., Quigley, K.S., Dickerson, B.C. \& Barrett, L.F. (2017) Evidence for a large-scale brain system supporting allostasis and interoception in humans. Nat. Hum. Behav., 1, 1-14. 
Kudielka, B.M., Hellhammer, D.H. \& Kirschbaum, C. (2007) Ten Years of Research with the Trier Social Stress Test-Revisited. Soc. Neurosci., 56, 83.

Lane, R., McRae, K., Reiman, E., Chen, K., Ahern, G. \& Thayer, J. (2009) Neural correlates of heart rate variability during emotion. NeuroImage, 44, 213-222.

Langewitz, W., Rüddel, H. \& Schächinger, H. (1994) Reduced parasympathetic cardiac control in patients with hypertension at rest and under mental stress. Am. Heart J. , 127, 122-128.

Liu, M.Y., Li, N., Li, W.A. \& Khan, H. (2017a) Association between psychosocial stress and hypertension: a systematic review and meta-analysis. Neurol. Res., 39, 573-580.

Liu, Y.Z., Wang, Y.X. \& Jiang, C.L. (2017b) Inflammation: the common pathway of stress-related diseases. Front. Hum. Neurosci., 11, 316.

MacKinnon, S., Gevirtz, R., McCraty, R. \& Brown, M. (2013) Utilizing heartbeat evoked potentials to identify cardiac regulation of vagal afferents during emotion and resonant breathing. Appl. Psychophysiol. Biofeedback, 38, 241-255.

Madsen, L.B., Rasmussen, J.K., Møller, D.S., Nyvad, O. \& Pedersen, E.B. (2008) Heart rate variability in white-coat hypertension. Blood Press. Monit., 13, 65-71.

Malliani, A.P., M.; Lombardi, F.; Cerutti, S. (1991) Cardiovascular neural regulation explored in the frequency domain. Circulation, 84, 482-492.

Mancia, G., De Backer, G., Dominiczak, A., Cifkova, R., Fagard, R., Germano, G., Grassi, G., Heagerty, A.M., Kjeldsen, S.E. \& Laurent, S. (2007) 2007 Guidelines for the management of arterial hypertension: The Task Force for the Management of Arterial Hypertension of 
the European Society of Hypertension (ESH) and of the European Society of Cardiology (ESC). Eur. Heart J., 28, 1462-1536.

Manly, B.F.J. (2006) Randomization, bootstrap and Monte Carlo methods in biology. Chapman and Hall/CRC.

McEwen, B.S. (1998) Protective and damaging effects of stress mediators. N. Engl. J. Med., 338, 171-179.

McEwen, B.S. (2007) Physiology and neurobiology of stress and adaptation: central role of the brain. Physiol. Rev., 87, 873-904.

Melloni, M., Billeke, P., Baez, S., Hesse, E., De la Fuente, L., Forno, G., Birba, A., GarcíaCordero, I., Serrano, C. \& Plastino, A. (2016) Your perspective and my benefit: multiple lesion models of self-other integration strategies during social bargaining. Brain, 139, 119.

Melloni, M., Sedeno, L., Hesse, E., Garcia-Cordero, I., Mikulan, E., Plastino, A., Marcotti, A., Lopez, J.D., Bustamante, C., Lopera, F., Pineda, D., Garcia, A.M., Manes, F., Trujillo, N. \& Ibanez, A. (2015) Cortical dynamics and subcortical signatures of motor-language coupling in Parkinson's disease. Sci Rep, 5, 11899.

Mocayar Marón, F.J., Ferder, L., Saraví, F.D. \& Manucha, W. (2019) Hypertension linked to allostatic load: from psychosocial stress to inflammation and mitochondrial dysfunction. Stress, 22, 169-181.

Muthukumaraswamy, S. (2013) High-frequency brain activity and muscle artifacts in MEG/EEG: a review and recommendations. Front. Hum. Neurosci., 7, 138. 
Nichols, T.E., Das, S., Eickhoff, S.B., Evans, A.C., Glatard, T., Hanke, M., Kriegeskorte, N., Milham, M.P., Poldrack, R.A. \& Poline, J.-B. (2017) Best practices in data analysis and sharing in neuroimaging using MRI. Nat. Neurosci., 20, 299.

Nichols, T.E. \& Holmes, A.P. (2002) Nonparametric permutation tests for functional neuroimaging: a primer with examples. Hum. Brain Mapp., 15, 1-25.

Nyklicek, I., Bosch, J.A. \& Amerongen, A.V. (2005) A generalized physiological hyperreactivity to acute stressors in hypertensives. Biol. Psych., 70, 44-51.

Oppenheimer, S., Kedem, G. \& Martin, W. (1996) Left-insular cortex lesions perturb cardiac autonomic tone in humans. Clinical Autonomic Research, 6, 131-140.

Osborne, J.W. \& Overbay, A. (2004) The power of outliers (and why researchers should always check for them). Pract. Assess. Res. Eval., 9, 6.

Pagani, M., Lombardi, F., Guzzetti, S., Rimoldi, O., Furlan, R., Pizzinelli, P., Sandrone, G., Malfatto, G., Dell'Orto, S. \& Piccaluga, E. (1986) Power spectral analysis of heart rate and arterial pressure variabilities as a marker of sympatho-vagal interaction in man and conscious dog. Circ. Res., 59, 178-193.

Pagani, M., Lombardi, F., Guzzetti, S., Sandrone, G., Rimoldi, O., Malfatto, G., Cerutti, S. \& Malliani, A. (1984) Power spectral density of heart rate variability as an index of sympatho-vagal interaction in normal and hypertensive subjects. J. Hypertens. Suppl. , 2 , S383.

Poldrack, R.A., Baker, C.I., Durnez, J., Gorgolewski, K.J., Matthews, P.M., Munafò, M.R., Nichols, T.E., Poline, J.-B., Vul, E. \& Yarkoni, T. (2017) Scanning the horizon: towards transparent and reproducible neuroimaging research. Nat. Rev. Neurosci., 18, 115. 
Pollatos, O., Kirsch, W. \& Schandry, R. (2005) Brain structures involved in interoceptive awareness and cardioafferent signal processing: a dipole source localization study. Hum. Brain Mapp., 26, 54-64.

Pollatos, O. \& Schandry, R. (2004) Accuracy of heartbeat perception is reflected in the amplitude of the heartbeat-evoked brain potential. Psychophysiology, 41, 476-482.

Rimmele, U., Seiler, R., Marti, B., Wirtz, P.H., Ehlert, U. \& Heinrichs, M. (2009) The level of physical activity affects adrenal and cardiovascular reactivity to psychosocial stress. Psychoneuroendocrinology, 34, 190-198.

Rimmele, U., Zellweger, B.C., Marti, B., Seiler, R., Mohiyeddini, C., Ehlert, U. \& Heinrichs, M. (2007) Trained men show lower cortisol, heart rate and psychological responses to psychosocial stress compared with untrained men. Psychoneuroendocrinology, 32, 627635.

Rohrmann, S., Hennig, J. \& Netter, P. (1999) Changing psychobiological stress reactions by manipulating cognitive processes. Int. J. Psychophysiol., 33, 149-161.

Ruediger, H., Seibt, R., Scheuch, K., Krause, M. \& Alam, S. (2004) Sympathetic and parasympathetic activation in heart rate variability in male hypertensive patients under mental stress. J. Hum. Hypertens., 18, 307-315.

Salamone, P.C., Esteves, S., Sinay, V.J., Garcia-Cordero, I., Abrevaya, S., Couto, B., Adolfi, F., Martorell, M., Petroni, A., Yoris, A., Torquati, K., Alifano, F., Legaz, A., Cassara, F.P., Bruno, D., Kemp, A.H., Herrera, E., Garcia, A.M., Ibanez, A. \& Sedeno, L. (2018) Altered neural signatures of interoception in multiple sclerosis. Hum. Brain Mapp., 39, 4743-4754. 
Salamone, P.C., Sedeño, L., Legaz, A., Bekinschtein, T., Martorell, M., Adolfi, F., FraileVazquez, M., Arriagada, N.R., Favaloro, L. \& Peradejordi, M. (2020) Dynamic neurocognitive changes in interoception after heart transplant. Brain Commun.

Sánchez, R.A., Ramos, F., Giannone, C., Fischer, P., Masnatta, L., Baglivo, H.P., Ramírez, A.J. \& Hollenberg, N.K. (2003) Parallel renal and extremity blood supply abnormalities in nonmodulation: responses to ACE inhibition. Hypertension, 41, 919-924.

Schandry, R. \& Montoya, P. (1996) Event-related brain potentials and the processing of cardiac activity. Biol. Psychol., 42, 75-85.

Schulz, A., Köster, S., Beutel, M.E., Schächinger, H., Vögele, C., Rost, S., Rauh, M. \& Michal, M. (2015) Altered patterns of heartbeat-evoked potentials in depersonalization/derealization disorder: neurophysiological evidence for impaired cortical representation of bodily signals. Psychosom. Med., 77, 506-516.

Schulz, A., Strelzyk, F., Ferreira de Sa, D.S., Naumann, E., Vogele, C. \& Schachinger, H. (2013) Cortisol rapidly affects amplitudes of heartbeat-evoked brain potentials--implications for the contribution of stress to an altered perception of physical sensations? Psychoneuroendocrinology, 38, 2686-2693.

Sedeno, L., Couto, B., Garcia-Cordero, I., Melloni, M., Baez, S., Morales Sepulveda, J.P., Fraiman, D., Huepe, D., Hurtado, E., Matallana, D., Kuljis, R., Torralva, T., Chialvo, D., Sigman, M., Piguet, O., Manes, F. \& Ibanez, A. (2016) Brain Network Organization and Social Executive Performance in Frontotemporal Dementia. J. Int. Neuropsychol. Soc., 22, 250-262.

Shaffer, F., McCraty, R. \& Zerr, C.L. (2014) A healthy heart is not a metronome: an integrative review of the heart's anatomy and heart rate variability. Front. Psychol., 5, 1040. 
Speilberger, C.D. \& Vagg, P.R. (1984) Psychometric properties of the STAI: a reply to Ramanaiah, Franzen, and Schill. Journal of personality assessment, 48, 95-97.

Steeb, B., García-Cordero, I., Huizing, M.C., Collazo, L., Borovinsky, G., Ferrari, J., Cuitiño, M.M., Ibáñez, A., Sedeño, L. \& García, A.M. (2018) Progressive compromise of nouns and action verbs in posterior cortical atrophy. Front. Psychol., 9, 1345.

Szczepanski, S.M., Crone, N.E., Kuperman, R.A., Auguste, K.I., Parvizi, J. \& Knight, R.T. (2014) Dynamic changes in phase-amplitude coupling facilitate spatial attention control in frontoparietal cortex. PLoS Biol., 12, e1001936.

Taelman, J., Vandeput, S., Spaepen, A. \& Van Huffel, S. (2009) Influence of Mental Stress on Heart Rate and Heart Rate Variability. IFMBE Proc., 22, 1366-1369.

Tarvainen, M.P., Niskanen, J.-P., Lipponen, J.A., Ranta-Aho, P.O. \& Karjalainen, P.A. (2014) Kubios HRV-heart rate variability analysis software. Comput. Methods Programs Biomed., 113, 210-220.

Task Force of the European Society of Cardiology and the North American Society of Pacing and Electrophysiology (1996) Heart rate variability: standards of measurement, physiological interpretation and clinical use. Circulation, 93, 1043-1065.

Thayer, J.F., Ahs, F., Fredrikson, M., Sollers, J.J., 3rd \& Wager, T.D. (2012) A meta-analysis of heart rate variability and neuroimaging studies: implications for heart rate variability as a marker of stress and health. Neurosci. Biobehav. Rev., 36, 747-756.

This article is protected by copyright. All rights reserved 
Thayer, J.F., Hansen, A.L., Saus-Rose, E. \& Johnsen, B.H. (2009) Heart rate variability, prefrontal neural function, and cognitive performance: the neurovisceral integration perspective on self-regulation, adaptation, and health. Ann. Behav. Med., 37, 141-153.

Thayer, J.F. \& Sternberg, E. (2006) Beyond heart rate variability: vagal regulation of allostatic systems. Ann. N. Y. Acad. Sci., 1088, 361-372.

Thayer, J.F., Yamamoto, S.S. \& Brosschot, J.F. (2010) The relationship of autonomic imbalance, heart rate variability and cardiovascular disease risk factors. Int. J. Cardiol., 141, 122-131.

Torralva, T., Roca, M., Gleichgerrcht, E., López, P. \& Manes, F. (2009) INECO Frontal Screening (IFS): A brief, sensitive, and specific tool to assess executive functions in dementiaCORRECTED VERSION. J. Int. Neuropsychol. Soc. , 15, 777-786.

Tzourio-Mazoyer, N., Landeau, B., Papathanassiou, D., Crivello, F., Etard, O., Delcroix, N., Mazoyer, B. \& Joliot, M. (2002) Automated anatomical labeling of activations in SPM using a macroscopic anatomical parcellation of the MNI MRI single-subject brain. NeuroImage, 15, 273-289.

Unger, T., Borghi, C., Charchar, F., Khan, N.A., Poulter, N.R., Prabhakaran, D., Ramirez, A., Schlaich, M., Stergiou, G.S. \& Tomaszewski, M. (2020) 2020 International Society of Hypertension global hypertension practice guidelines. Hypertension, 75, 1334-1357.

Vlcek, M., Radikova, Z., Penesova, A., Kvetnansky, R. \& Imrich, R. (2008) Heart rate variability and catecholamines during hypoglycemia and orthostasis. Auton. Neurosci., 143, 53-57.

Wei, L., Chen, H. \& Wu, G.-R. (2018) Heart rate variability associated with grey matter volumes in striatal and limbic structures of the central autonomic network. Brain Res., 1681, 14-20. 
WHO (1992) The ICD-10 classification of mental and behavioural disorders: clinical descriptions and diagnostic guidelines. World Health Organization.

Wiener, A., Rohr, C.S., Naor, N., Villringer, A. \& Okon-Singer, H. (2020) Emotion Regulation in Essential Hypertension: Roles of Anxiety, Stress, and the Pulvinar. Front. Behav. Neurosci.

Wirtz, P.H., von Kanel, R., Mohiyeddini, C., Emini, L., Ruedisueli, K., Groessbauer, S. \& Ehlert, U. (2006) Low social support and poor emotional regulation are associated with increased stress hormone reactivity to mental stress in systemic hypertension. J. Clin. Endocrinol. Metab., 91, 3857-3865.

Xhyheri, B., Manfrini, O., Mazzolini, M., Pizzi, C. \& Bugiardini, R. (2012) Heart rate variability today. Prog. Cardiovasc. Dis., 55, 321-331.

Yamakawa, K., Matsunaga, M., Isowa, T., Kimura, K., Kasugai, K., Yoneda, M., Kaneko, H. \& Ohira, H. (2009) Transient responses of inflammatory cytokines in acute stress. Biological psychology, 82, 25-32.

Yoris, A., Abrevaya, S., Esteves, S., Salamone, P., Lori, N., Martorell, M., Legaz, A., Alifano, F., Petroni, A., Sanchez, R., Sedeno, L., Garcia, A.M. \& Ibanez, A. (2018) Multilevel convergence of interoceptive impairments in hypertension: New evidence of disrupted body-brain interactions. Hum. Brain Mapp., 39, 1563-1581.

Yoris, A., Garcia, A.M., Traiber, L., Santamaria-Garcia, H., Martorell, M., Alifano, F., Kichic, R., Moser, J.S., Cetkovich, M., Manes, F., Ibanez, A. \& Sedeno, L. (2017) The inner world of overactive monitoring: neural markers of interoception in obsessive-compulsive disorder. Psychological medicine, 47, 1957-1970. 
Zimmerman, D.W. (1994) A note on the influence of outliers on parametric and nonparametric tests. J. Gen. Psychol., 121, 391-401.

\section{Tables}

Table 1. Demographic, cardiovascular, cognitive and emotional measures

\begin{tabular}{|c|c|c|c|}
\hline \multirow{2}{*}{ Variables } & \multicolumn{2}{|l|}{ Groups } & \multirow{2}{*}{ Statistics } \\
\hline & Controls & $\begin{array}{c}\text { HTD } \\
\text { patients }\end{array}$ & \\
\hline \multicolumn{4}{|l|}{ Demographic results } \\
\hline Gender (F:M) & 18:09 & $13: 12$ & $\chi^{2}=0.63, P=0.43$ \\
\hline Age & $66.04(7.29)$ & $67.56(9.34)$ & $\begin{array}{l}F_{1,50}=0.43, P=0.51, \eta p^{2}= \\
0.00\end{array}$ \\
\hline Education & $16.59(2.39)$ & $15.00(4.54)$ & $\begin{array}{l}F_{1,50}=2.55, P=0.12, \eta p^{2}= \\
0.04\end{array}$ \\
\hline Body mass index & $25.62(3.37)$ & $26.89(3.45)$ & $\begin{array}{l}F_{1,50}=1.78, P=0.19, \eta p^{2}= \\
0.03\end{array}$ \\
\hline Handedness (R:L) & $26: 0$ & $23: 01$ & --- \\
\hline \multicolumn{4}{|c|}{ Clinical cardiovascular assessment } \\
\hline ABPM (systolic 24hrs.) & $\begin{array}{l}114.47 \\
(18.21)\end{array}$ & $\begin{array}{l}127.60 \\
(13.25)\end{array}$ & $\begin{array}{l}F_{1,37}=6.67, P=0.01^{*}, \eta p^{2}= \\
0.15\end{array}$ \\
\hline OBP systolic & 132.85 & 143.51 & $F_{1,33}=4.19, P=0.04^{*}, \eta p^{2}=$ \\
\hline
\end{tabular}




\begin{tabular}{|l|l|l|l|}
\hline & $(13.10)$ & $(17.74)$ & 0.11 \\
\hline OBP diastolic & $77.68(6.99)$ & $82.31(4.92)$ & $\begin{array}{l}F_{1,31}=4.46, P=0.04^{*}, \eta p^{2}= \\
0.12\end{array}$ \\
\hline
\end{tabular}

\section{Cognitive symptom assessment}

\begin{tabular}{|l|l|l|l|}
\hline IFS & $25.26(2.35)$ & $23.95(3.68)$ & $\begin{array}{l}F_{1,48}=2.27, P=0.13, \eta p^{2}= \\
0.04\end{array}$ \\
\hline
\end{tabular}

\section{Emotional symptom assessment}

\begin{tabular}{|l|l|l|l|}
\hline BDI - II & $11.47(9.10)$ & $9.38(4.66)$ & $\begin{array}{l}F_{1,32}=0.58, P=0.450, \eta p^{2}= \\
0.18\end{array}$ \\
\hline
\end{tabular}

Results are presented as mean $(S D)$. The asterisk indicates significant differences. Demographic and clinical data were assessed through ANOVAs. Gender was analyzed with Pearson's chi-squared $\left(\chi^{2}\right)$ test. Effects sizes were calculated through partial eta $\left(\eta p^{2}\right)$. ABPM: ambulatory blood pressure monitor; BDI-II: Beck Depression Inventory - II; IFS: INECO Frontal Screening; OBP: office blood pressure.

\section{Table 2. State-anxiety during TSST}

\begin{tabular}{|l|l|l|l|}
\hline Groups & \multicolumn{2}{|l|}{ Variables } & Statistics \\
\hline & $\begin{array}{l}\text { State- } \\
\text { anxiety } \\
\text { baseline }\end{array}$ & $\begin{array}{l}\text { State- } \\
\text { anxiety } \\
\text { stress }\end{array}$ & \\
\hline All groups & $27.05(6.31)$ & $31.16(8.26)$ & $t_{47}=-3.27, P=0.002^{*}, d=0.47$ \\
\hline Controls & $28.03(4.53)$ & $30.03(6.74)$ & $t_{25}=-1.44, P=0.162, d=0.28$ \\
\hline HTD patients & $26.86(7.98)$ & $32.50(9.75)$ & $t_{21}=-3.21, P=0.004^{*}, d=0.68$ \\
\hline
\end{tabular}

State-anxiety was assessed through the state scale of the State-Trait Anxiety Inventory (STAI) (Speilberger \& Vagg, 1984). Results are presented as mean $(S D)$. The asterisk indicates significant difference. Effect sizes were calculated through Cohen's d. HTD: Hypertensive disease. 
Table 3. Heart rate during TSST

\begin{tabular}{|l|l|l|l|}
\hline Groups & \multicolumn{2}{|l|}{ Variables } & Statistics \\
\hline & $\begin{array}{l}\text { HR } \\
\text { baseline }\end{array}$ & $\begin{array}{l}\text { HR } \\
\text { stress }\end{array}$ & \\
\hline Controls & 65.46 & 72.73 & $t_{24}=-7.67, P<0.001^{*}, d=1.53$ \\
$(5.15)$ & $(5.24)$ & \\
\hline HTD patients & $\begin{array}{l}68.73 \\
(9.13)\end{array}$ & $\begin{array}{l}84.19 \\
(21.43)\end{array}$ & $t_{20}=-3.28, P=0.004^{*}, d=0.71$ \\
\hline
\end{tabular}

Results are presented as mean (SD). The asterisk indicates significant differences. Effect sizes were calculated through Cohen's d. HTD: Hypertensive disease; HR: Heart rate.

Table 4. Regression between GM in fronto-temporo-insular regions and HRV during the TSST

\begin{tabular}{|l|l|l|l|l|l|}
\hline Brain region & Peak $\boldsymbol{t}$ & $\begin{array}{l}\text { Peak P } \\
\text { (uncl) }\end{array}$ & $\boldsymbol{x}$ & $\boldsymbol{y}$ & $\boldsymbol{z}$ \\
\hline Controls - TSST baseline & 5.53 & $<0.001$ & 37.5 & 57 & -1.5 \\
\hline Middle orbitofrontal R & 5.46 & $<0.001$ & 42 & 51 & -9 \\
\hline & 4.63 & $<0.001$ & 42 & 46.5 & -18 \\
\hline & 5.27 & $<0.001$ & -45 & 42 & -18 \\
\hline Insula L & 4.78 & $<0.001$ & -42 & 4.5 & -1.5 \\
\hline Controls - TSST stress & \multicolumn{5}{|l|}{} \\
\hline Putamen R & 5.29 & $<0.001$ & 28.5 & 18 & 6 \\
\hline
\end{tabular}




\begin{tabular}{|l|l|l|l|l|l|}
\hline & 4.56 & $<0.001$ & 34.5 & 25.5 & 10.5 \\
\hline & 4.20 & $<0.001$ & 42 & 10.5 & 12 \\
\hline Inferior orbitofrontal R & 4.35 & $<0.001$ & 49.5 & 33 & -6 \\
\hline Superior orbitofrontal R & 4.28 & $<0.001$ & 27 & 58.5 & 0 \\
\hline
\end{tabular}

HTD patients - TSST baseline

Middle frontal L

5.17

$<0.001$

$-39$

24

49.5

Regressions between GM volume and HRV during TSST: No associations between GM volume and HRV during TSST stress were found in HTD patients. HTD: Hypertensive disease; R: Right; L: Left.

\section{Figure captions}

Figure 1. (a) Differences in HRV during the TSST: Baseline and stress periods (superior row), subtraction between stress and baseline (inferior row). Significant differences are indicated with an asterisk $(P<0.05)$. Individual modulations are represented inside and outside the box as points. The middle box line indicates the group's mean values. (b) HEP modulation in frontal ROI during the baseline and stress periods during the TSST: Differences between groups were calculated via Monte Carlo permutations analyses (5000 permutations, $P<0.05$ ), point by point (Manly, 2006). Following previous reports, a minimum extension of five consecutive points was establish to ensure temporally robust results (García-Cordero et al., 2016; Salamone et al., 2018). Shadowed bars around ERP modulations indicate SEM. Grey boxes indicate significant differences. (c) Association between HEP modulation and HRV during the baseline and stress periods of the TSST: Significant correlations are indicated with an asterisk $(P<0.05)$. (d) Regressions between GM volume and HRV during the baseline and stress periods of the TSST: these analyses were conducted to identify regions in each group that were associated with the HRV during the TSST $(P<0.001$ uncorrected, extent threshold $=30$ voxels). Baseline (left), 
stress (right). HEP: Heart evoked potential; HRV: Heart rate variability; HTD: Hypertensive disease; TSST: Trier Stress Social Test.

This article is protected by copyright. All rights reserved 
(a) Differences in HRV

Controls if Patients
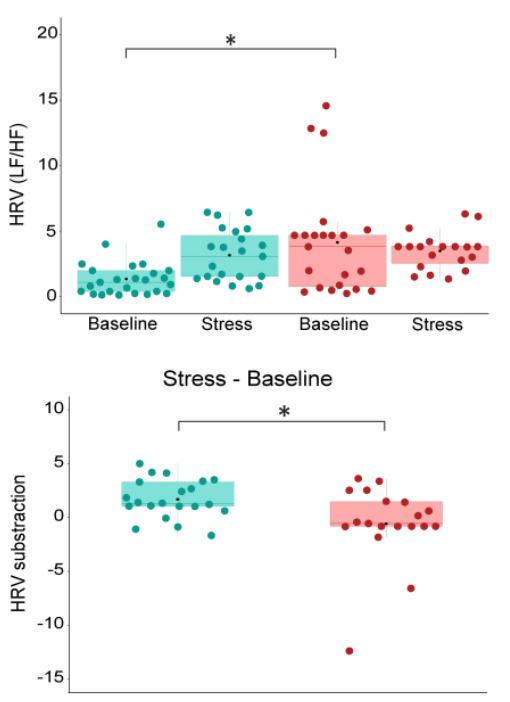

d - Gray matter volume associated to HRV

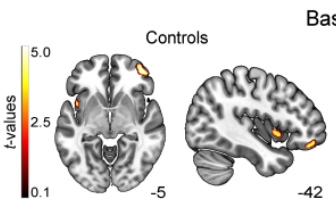

(b) HEP modulation

EControls $=$ Patients

Baseline
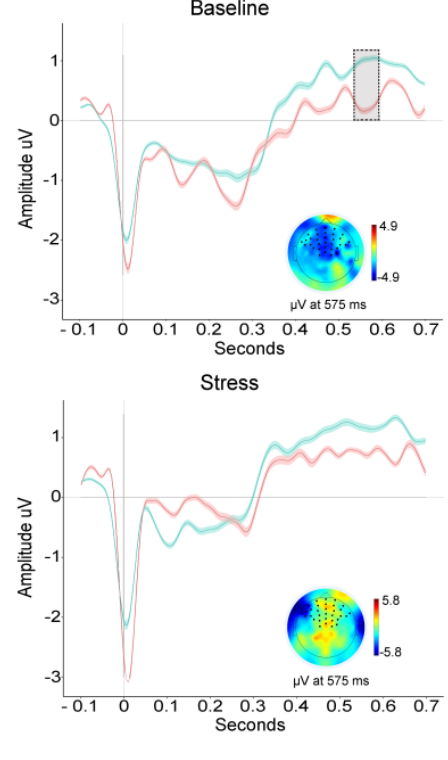

(c) HEP and HRV association

- Controls $\leftarrow$ Patients
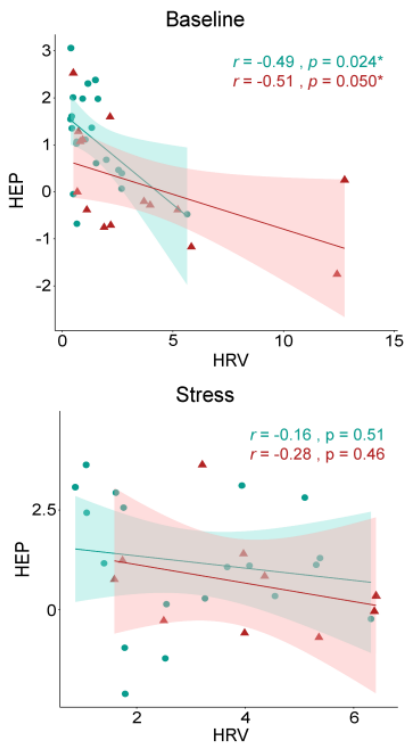

Stress Patients
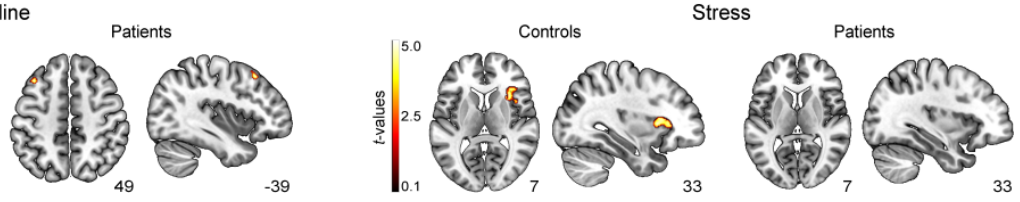

ejn_14979_f1.tif

This article is protected by copyright. All rights reserved 